\title{
Integrated FE-LE Modelling Method For a Simplified Balanced-Armature Receiver
}

\author{
Wei Sun and Wenxiang Hu \\ Institute of Acoustics, Tongji University, 1239 Siping Road, Shanghai, P.R. China 200092.
}

(Received 5 May 2016; accepted 27 October 2016)

The conventional lumped-element (LE) method is insufficient in modelling the wideband balanced-armature receiver (BAR) when only the fundamental mode of the vibration system is considered. To overcome this limitation, we propose an integrated FE-LE modelling method, i.e. the finite-element (FE) modelled mechanical domain integrated with the LE modelled acoustic domain or vice versa. This method makes use of the FE method to implement the multimode modelling of the more interested physical domain and the LE method to deal with the remaining features, in which they are bidirectionally coupled. The coupling relations between the involved physical domains are explicitly defined. In addition, the insufficient predication of the LE model is preliminarily investigated in terms of the first two vibration modes of the mechanical domain. Numerical results show that the proposed method is far superior to the commonly used LE method in accuracy and to the full FE method in efficiency.

\section{Nomenclature}

$F_{e}$

$k_{m}$

$m_{m}$

$r_{m}$

$\mathrm{C}_{a, f v}$

$\mathrm{C}_{a, r v}$

$C_{a, 2 c c}$

$M_{a, f v}$

$M_{a, t u b e}$

$q_{a, m e m}$

$v_{m, \text { mem }}$

$v_{m, M P}$

$A_{\text {mem,eff }}$

$d A_{\text {mem }}$

$A_{\text {mem }}$

$F_{m, m e m}$

$P_{f v}$

$P_{r v}$

$p_{f v}$

$p_{r v}$

$p$

n

$\rho_{0}$

$a_{n}$

$a_{a, m e m}$

j

$\omega$

$r_{\text {mem }}$

$f_{0}$

$C_{a}$

$M_{a}$

$V_{0}$

$c_{0}$ $l$

S

driving force at DP

stiffness of mechanical domain with respect to DP $l_{e f f}$

moving mass of mechanical domain

damping ratio of mechanical domain

acoustic compliance of front volume

acoustic compliance of rear volume

acoustic compliance of the $2 \mathrm{cc}$ acoustic coupler

acoustic mass of front volume

acoustic mass of tube

induced volume velocity by membrane

distributed velocity of membrane

velocity at MP

effective area of membrane

area element of membrane

actual area of membrane

induced force over membrane

induced lumped pressure in front volume

induced lumped pressure in rear volume

induced distributed pressure in front volume

induced distributed pressure in rear volume

acoustic pressure

normal vector

air density

inward normal acceleration

equivalent acceleration loaded over the coupling interface of acoustic domain

imaginary unit

angular velocity

ratio between the effective area and the realistic area of membrane

fundamental resonance of mechanical system

acoustic compliance

acoustic mass

static volume of air volume

sound velocity of air actual length of slit or tube

sectional area (vertical to the length direction) of slit or tube

length of slit or tube with end correction radius of sound outlet or tube

\section{INTRODUCTION}

Balanced-armature receiver (BAR), predominantly used in hearing aids and increasingly trended in high-end earphone, is inherently a nonlinear transducer for its relatively complicated mechanical structure as well as multi-physics domains contained. Owing to the increasing demand for high-fidelity music sound, the wideband BAR incorporating the multiple modes of the vibration system needs to be developed. However, the currently used lumped-element (LE) models ${ }^{1-7}$ are no longer applicable due to the lack of high-order modes of the vibration system.

Until now, only limited studies were dedicated to BAR. In the earlier studies, Hunt discussed the basic principle of BAR ${ }^{1}$ and Henning designed a smaller but more sensitive BAR for a telephone set. ${ }^{2}$ Later, Kampinga et al. fulfilled a study about the visco-thermal air layer influence on the hearing aids transducer. ${ }^{3}$ Jensen et al. created a nonlinear time-domain model to predict the nonlinear factors from both the mechanical and electromagnetic domains. ${ }^{4}$ More recently, Kim et al. established a two-port network analysis considering two key elements, semi-inductor and gyrator, which are, respectively, used to more accurately approximate the electromagnetic eddy-current and anti-reciprocity characteristic of the BAR. ${ }^{5}$ Tsai et al. studied the asymmetrical magnetic flux influence on the nonlinear harmonic distortion. ${ }^{6}$ A recent $\mathrm{PhD}$ study by Jensen gained more insights into the nonlinear distortion mechanisms and efficiency in modelling the BAR. ${ }^{7}$ However, all of these studies are based on the fundamental mode of the vibration system. For this reason, we launch the study of modelling the wideband multimode BAR. 


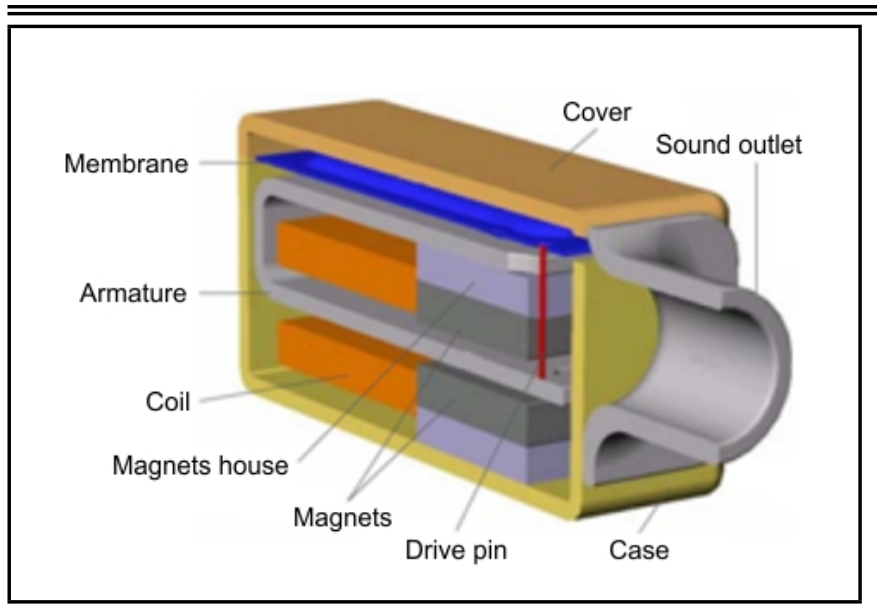

Figure 1. Sectional view of a BAR (Courtesy of Sonion A/S).

It is known that the finite-element (FE) method is much accurate and adaptive but extremely time-consuming and even impracticable sometimes, whereas the LE method is somewhat simplified but particularly efficient. The LE method concerning merely the fundamental modes of the contained physical domains is normally used to model electroacoustic transducers. However, it may be no longer applicable if multiple modes of the system are considered or more accurate prediction is required. Although the full FE modelling method is widely applicable, it is rather complicated and time-consuming.

The integrated FE-LE modelling method presented in this study is to model the BAR, which is capable of containing the multiple modes of the system. It has the advantages of both the FE method in accuracy and the LE method in efficiency. Unlike the methods commonly used, e.g. FE-aided LE method ${ }^{8}$ or full FE method, ${ }^{9,10}$ the developed method is to integrate the FE modelled domain with the LE modelled domain seamlessly via the commercial FE software. In the modelling, the FE part is capable of including the multiple modes of the modelled domain.

\section{DEFINITION OF BAR MODEL}

\subsection{Basic Principles of BAR}

As shown in Fig. 1, BAR is named for its magnetically balanced position of the armature between the two magnets. Therefore, the principles of all these types of transducers are similar. It mainly consists of coil, armature, drive pin, membrane, cover, case, permanent magnets, magnetic house, and sound outlet. When there is an electric current through the coil, it magnetizes the armature one way or the other. Then the magnetized armature starts to vibrate because of its interaction with the magnets. The moving armature then drives the membrane by the connected drive pin. Sound is subsequently produced in the front volume (the cavity between membrane and cover) by the vibrating membrane, and finally transmitted through the sound outlet. The sealed rear volume between the membrane and the case works like a spring to stiffen the system as required.

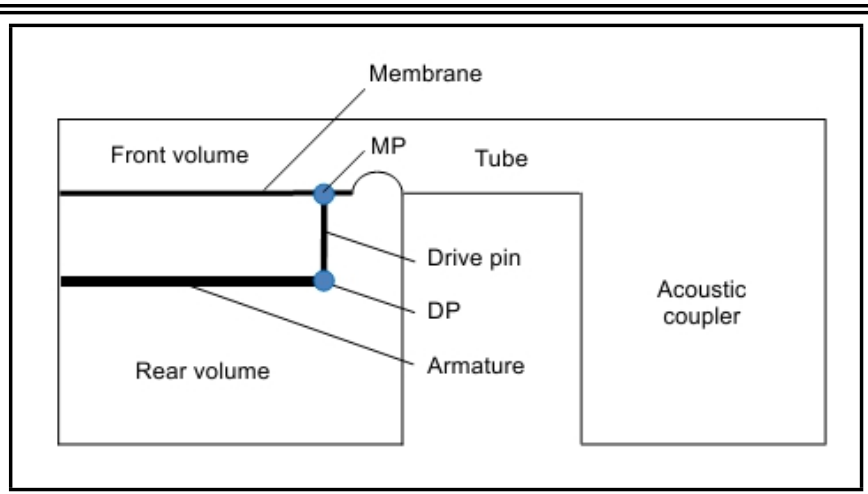

Figure 2. Schematic graph of a simplified BAR model containing only the mechanical and acoustic domains, in which the drive point (DP) is the connection point between armature and drive pin and the measure point (MP) is the connection point between drive pin and membrane.

\subsection{Simplified BAR Model}

The purpose of this work is to evaluate the integrated FELE modelling method for a simplified BAR. As illustrated in Fig. 2, it contains only the mechanical domain (armature, drive pin, and membrane) and the acoustic domain (front volume, rear volume, sound tube, and acoustic coupler). The electromagnetic domain and its transduction relation to the mechanical domain are out of the scope of this study, and the acoustic damping is excluded as well. Magnetic force of BAR is normally loaded over the magnet projecting areas on the armature. It can be equivalent to a point force around the centre of the areas, i.e. force point, in the LE modelling. This point force can be further equivalent to a force at drive point (DP) in the linear vibrating range of the system. With these assumptions, a constant point force is applied at DP to replace the magnetic force in this study. To make modelling more reliable, the mechanical structure of BAR is treated as a whole to determine its LE parameters.

\section{DEVELOPMENT OF INTEGRATED FE-LE MODELS FOR BAR}

According to the literatures, ${ }^{1,2}$ a mechanical-acoustic equivalent circuit (Fig. 3), i.e. LE model, is built for the defined BAR in Fig. 2. In the circuit, a constant driving force $F_{e}$ applied at DP is equivalent to an alternating voltage source; mechanical stiffness $k_{m}$, mass $m_{m}$, and damping $r_{m}$ of the entire mechanical structure are equivalent to electric capacitance, inductance, and resistance, respectively. For the acoustic domain, acoustic compliances (front volume $C_{a, f v}$, rear volume $C_{a, r v}$, and the $2 \mathrm{cc}$ acoustic coupler $C_{a, 2 c c}$ ) are equivalent to electric capacitances, and acoustic masses (front volume $M_{a, f v}$, tube $\left.M_{a, t u b e}\right)$ are equivalent to electric inductances. The flowing variable $v_{m, M P}$ in the mechanical domain is the velocity at the measure point (MP), i.e. the connection point between the drive pin and the membrane, and $q_{a, m e m}$ in the acoustic domain is the volume velocity induced by the membrane. Further, $P_{f v}$ and $P_{r v}$ are, respectively, the pressures in the front and rear volumes. 


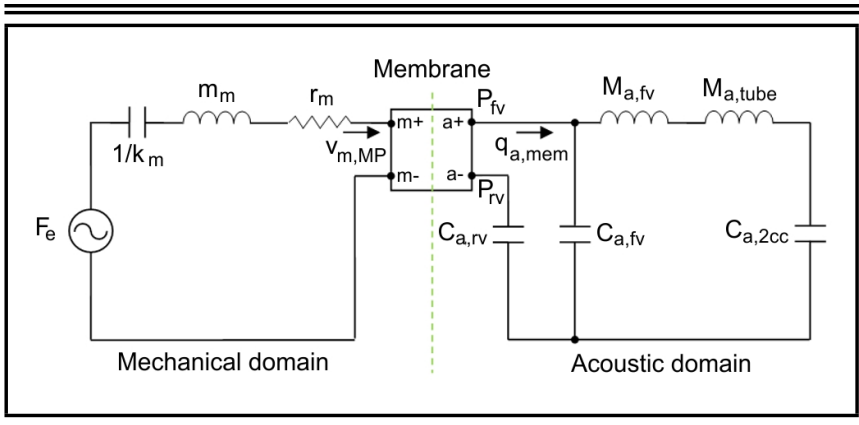

Figure 3. LE model for the defined BAR.

\subsection{Development of Two Integrated FE-LE Models}

The LE model as shown in Fig. 3 is normally used to evaluate electroacoustic transducers. However, the LE parameters of the contained physical domains used are merely the approximations with respect to the fundamental modes of the system. It is incapable of describing the system if higher-order modes of the system are concerned or more accurate prediction is required. Therefore, an improved model capable of incorporating the higher-order modes of the system is necessary to be developed. However, it is very complicated to build such a model using the LE method only. We therefore propose an integrated FE-LE modelling method to resolve this problem. This method has the advantages of both the FE and LE methods and is also convenient to be developed with the relevant software.

The mechanical and acoustic domains contained in the BAR may be modelled using either FE or LE method. Therefore, there are two integrated models, i.e. the integrated FE mechanical with LE acoustic (MechaFE-AcoLE) model and the integrated LE mechanical with FE acoustic (MechaLE-AcoFE) model. Generally, the FE method is preferably used to model the more concerned domain, whereas the LE method is used to model the remaining features.

\subsubsection{Integrated MechaFE-AcoLE model}

If the special concern is given to the mechanical domain, the integrated MechaFE-AcoLE model (Fig. 4) can be established for the defined BAR. In the model, the mechanical domain is modelled with the FE method and the acoustic domain is modelled with the LE method. The two domains are bidirectionally coupled via the membrane. Specifically, the membrane transfers its vibrating velocity $v_{m, m e m}$ to the air in the front and rear volumes with the induced volume velocity $q_{a, m e m}$ defined in Eq. (1); and meanwhile, the acoustic pressures generated in the volumes impose back on the membrane with the generated force $F_{m, m e m}$ defined in Eq. (2).

$$
\begin{gathered}
q_{a, m e m}=\left\{\begin{array}{c}
A_{m e m, e f f} v_{m, M P} \\
\iint v_{m, m e m} d A_{m e m}
\end{array} ;\right. \\
F_{m, m e m}=\left\{\begin{array}{c}
\left(P_{f v}-P_{r v}\right) A_{m e m, e f f} \\
\iint\left(p_{f v}-p_{r v}\right) d A_{m e m}
\end{array} ;\right.
\end{gathered}
$$

where $A_{m e m, e f f}$ is the effective area of the membrane, $v_{m, M P}$ is the velocity at MP, $d A_{m e m}$ is the area element of the membrane; $P_{f v}$ and $P_{r v}$ are the lumped pressures, $p_{f v}$ and $p_{r v}$ are

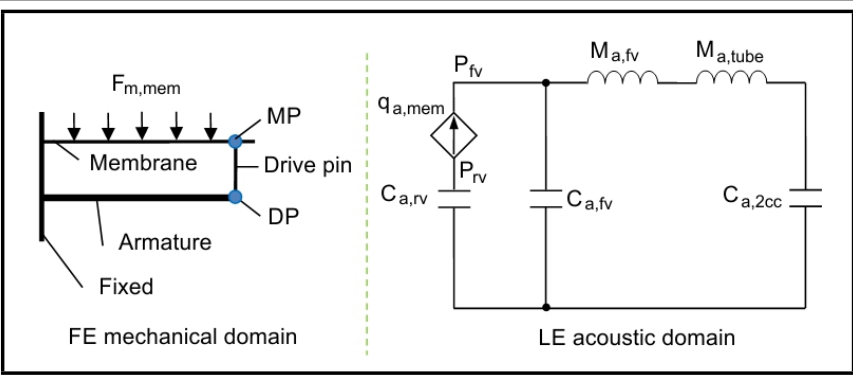

Figure 4. Integrated MechaFE-AcoLE model for the defined BAR.

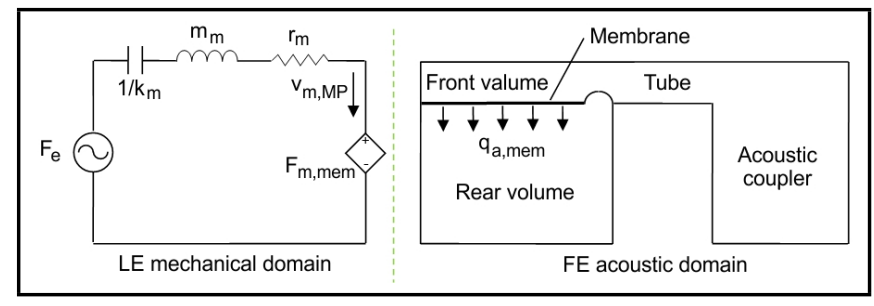

Figure 5. Integrated MechaLE-AcoFE model for the defined BAR

the distributed pressures in the front and rear volumes, respectively; and in Eqs. (1) and (2), the lumped formulae are applicable to the LE modelling and the distributed formulae are applicable to the FE modelling.

\subsubsection{Integrated MechaLE-AcoFE model}

Afterwards, the integrated MechaLE-AcoFE model (Fig. 5) is built by exchanging the two concerned domains in order to accurately model the acoustic domain.

The coupling relations between the two domains here are theoretically similar to the above model. The only difference is that there is no direct coupling variable from the LE mechanical domain to the FE acoustic domain. As an alternative, the coupling is realized with Neumann (or second-type) boundary condition $^{11,12}$

$$
-\mathbf{n} \cdot\left(-\frac{\mathbf{1}}{\rho_{\mathbf{0}}}\right) \nabla \mathbf{p}=\mathbf{a}_{\mathbf{n}}
$$

where $\mathbf{n}$ is the normal vector, $\rho_{0}$ is the air density, $p$ is the acoustic pressure, and $a_{n}$ is the inward normal acceleration.

Specifically, the acceleration can be expressed as

$$
a_{a, m e m}=j \omega v_{m, M P} r_{m e m} ;
$$

where $a_{a, m e m}$ is the equivalent acceleration loaded over the coupling interface of the acoustic domain, $j$ is the imaginary unit, $\omega$ is the angular velocity, and $r_{m e m}=A_{m e m, e f f} / A_{m e m}$ is the ratio of the effective area to the actual area $A_{m e m}$ of the membrane.

To sum up, the coupling relations for the two integrated models are explicitly indicated in Fig. 6.

\subsection{Determination of the LE Parameters}

The mechanical domain of BAR consisting of balanced armature, membrane, and drive pin plays an important role in its overall performance. As discussed above, the corresponding LE parameters are determined by treating the mechanical domain as a whole. Given an arbitrary mechanical structure 


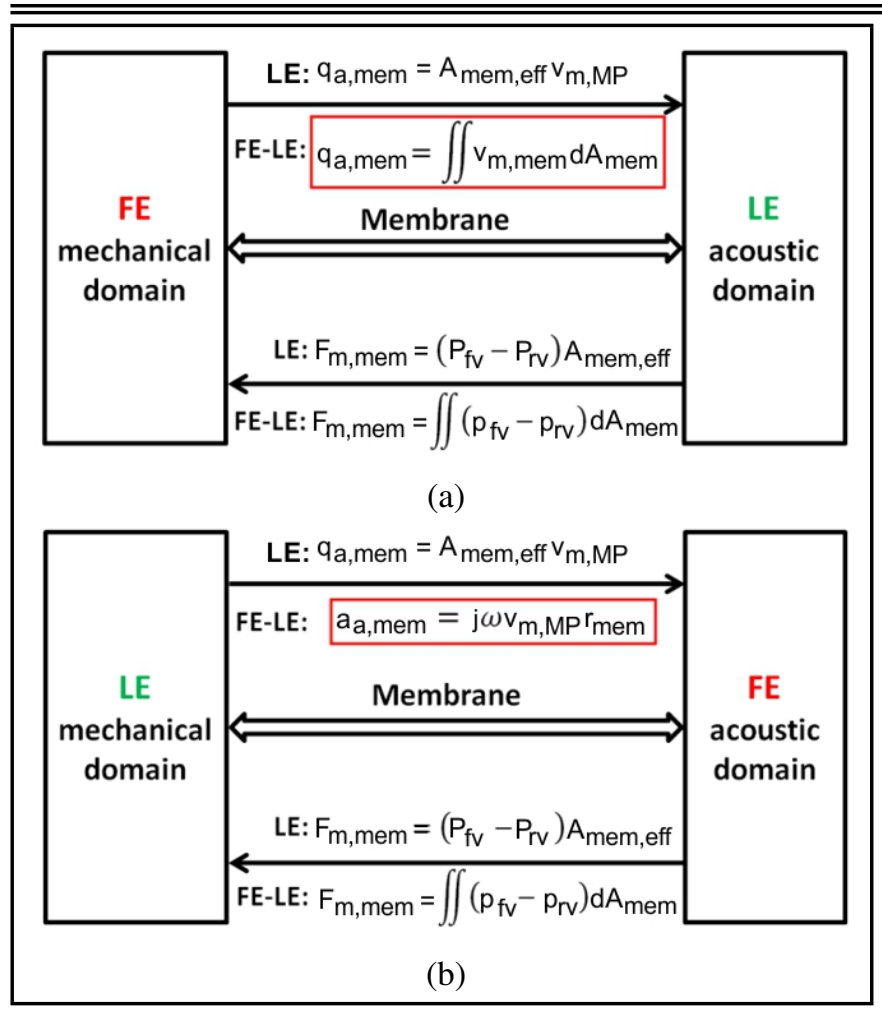

Figure 6. Coupling relations of the two integrated models: (a) integrated MechaFE-AcoLE model; (b) integrated MechaLE-AcoFE model.

of BAR, its fundamental resonance $f_{0}$ and stiffness $k_{m}$ at MP can be easily computed with the aid of finite element software. The corresponding moving mass $m_{m}$ is then determined by ${ }^{13}$

$$
m_{m}=\frac{k_{m}}{\left(2 \pi f_{0}\right)^{2}} \text {. }
$$

The LE parameters for the acoustic domain are determined based on the assumption of ideal air. Acoustic compliance $C_{a}$ and mass $M_{a}$ of acoustic component are, respectively, calculated with ${ }^{14}$

$$
C_{a}=\frac{V_{0}}{\rho_{0} c_{0}^{2}}
$$

and

$$
M_{a}=\frac{\rho_{0} l_{e f f}}{S}
$$

where $V_{0}$ is the static volume of air cavity, $\rho_{0}$ and $c_{0}$ are, respectively, the density and the sound speed of air under normal temperature; $l_{\text {eff }}=l+8 r /(3 \pi)$ is the effective length including the end correction, $l$ is the actual length, $S$ is the sectional area (vertical to the length direction) of slit or tube and $r$ is the radius of tube.

\section{NUMERICAL CALCULATION AND ANALYSIS}

\subsection{Model Definition}

Table 1 lists all the material properties for the following exemplified BAR sample.

\subsubsection{Definition of a simplified BAR model}

Figure 7 shows a BAR sample as defined in Fig. 2. Dimensions of the mechanical components are, respectively, given as follows: armature $5 \times 1.50 \times 0.10 \mathrm{~mm}^{3}$
Table 1. Material properties.

\begin{tabular}{ccccc}
\hline Component & Material type & $\begin{array}{c}\text { Density } \\
\left(\mathrm{kg} / \mathrm{m}^{3}\right)\end{array}$ & $\begin{array}{c}\text { Young's } \\
\text { modulus }(\mathrm{Pa})\end{array}$ & $\begin{array}{c}\text { Poisson's } \\
\text { ratio }\end{array}$ \\
\hline Membrane & Aluminum & 2,700 & $0.70 \times 10^{11}$ & 0.35 \\
$\begin{array}{c}\text { Drive pin } \\
\text { Armature }\end{array}$ & Copper & 8,960 & $1.20 \times 10^{11}$ & 0.34 \\
$\begin{array}{c}\text { Volumes, tube, } \\
\text { and coupler }\end{array}$ & Air & 1.20 & $\begin{array}{c}344(\mathrm{~m} / \mathrm{s}) \\
\text { (sound speed) }\end{array}$ & - \\
\hline
\end{tabular}

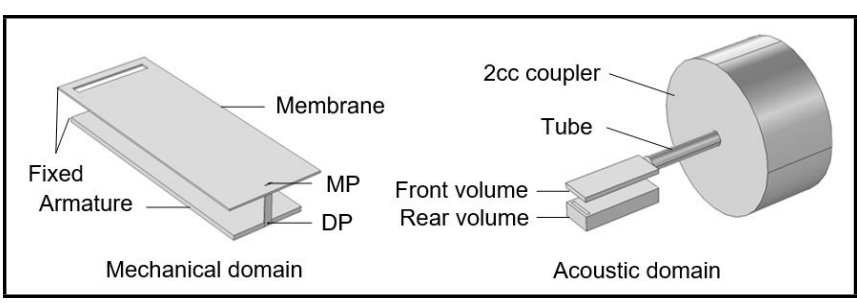

Figure 7. Geometry of the exemplified BAR sample.

(length $\times$ width $\times$ thickness), drive pin $0.70 \times 0.15 \times 0.025 \mathrm{~mm}^{3}$ and membrane $5.20 \times 2 \times 0.04 \mathrm{~mm}^{3}$ with a centralized rectangular hole $\left(1.60 \times 0.20 \times 0.025 \mathrm{~mm}^{3}, 0.20 \mathrm{~mm}\right.$ away from the fixed boundary). In reality, the membrane consists of metal and foil layers. Here, we consider only the dominant metal layer, but the two volumes are still completely isolated over the hole in our study. The two acoustic volumes, front volume $3.12 \times 10^{-9} \mathrm{~mm}^{3}\left(5.20 \times 2 \times 0.30 \mathrm{~mm}^{3}\right)$ and sealed rear volume $10 \times 10^{-9} \mathrm{~mm}^{3}\left(5.20 \times 2 \times 0.96 \mathrm{~mm}^{3}\right)$, are isolated by the membrane. The right side of the front volume is connected to a tube with length $5 \mathrm{~mm}$ and diameter $1 \mathrm{~mm}$, and a $2 \mathrm{cc}$ acoustic coupler is connected thereafter. A constant force with amplitude $1 \times 10^{-3} N$ is loaded at DP. Variables to be evaluated are the velocity at MP and the sound pressure level (SPL) in the 2cc coupler, which is obtained by averaging the sound pressure over the bottom surface of the $2 \mathrm{cc}$ coupler (i.e. right side of the 2cc coupler).

\subsubsection{Four models concerned for comparison}

Four models, the LE model concerning only the fundamental modes of the included physical domains, the full FE model and the two integrated FE-LE models, are built to evaluate the proposed integrated modelling method in our study. The FE model is used as a reference. The LE model in Fig. 3 is performed with PSpice, and the FE model is built in Comsol Multiphsics 3.5a using the acoustic and mechanical modules. The two integrated models, shown in Figs. 4 and 5, are also established in Comsol Multiphysics 3.5a with the spice, mechanical, and acoustic modules. Frequency-domain analyses are performed for all the four models, and the frequency of interest is ranged from $20 \mathrm{~Hz}$ to $20 \mathrm{kHz}$ with 100 points per decade.

According to Eqs. (5)-(7), the determined LE parameters are listed in Table 2.

\subsection{Numerical Results and Analyses}

To begin with, the concerned four models are compared in terms of the computation time. Then the two integrated models are, respectively, evaluated by referring to both the FE and LE 
W. Sun, et al.: INTEGRATED FE-LE MODELLING METHOD FOR A SIMPLIFIED BALANCED-ARMATURE RECEIVER

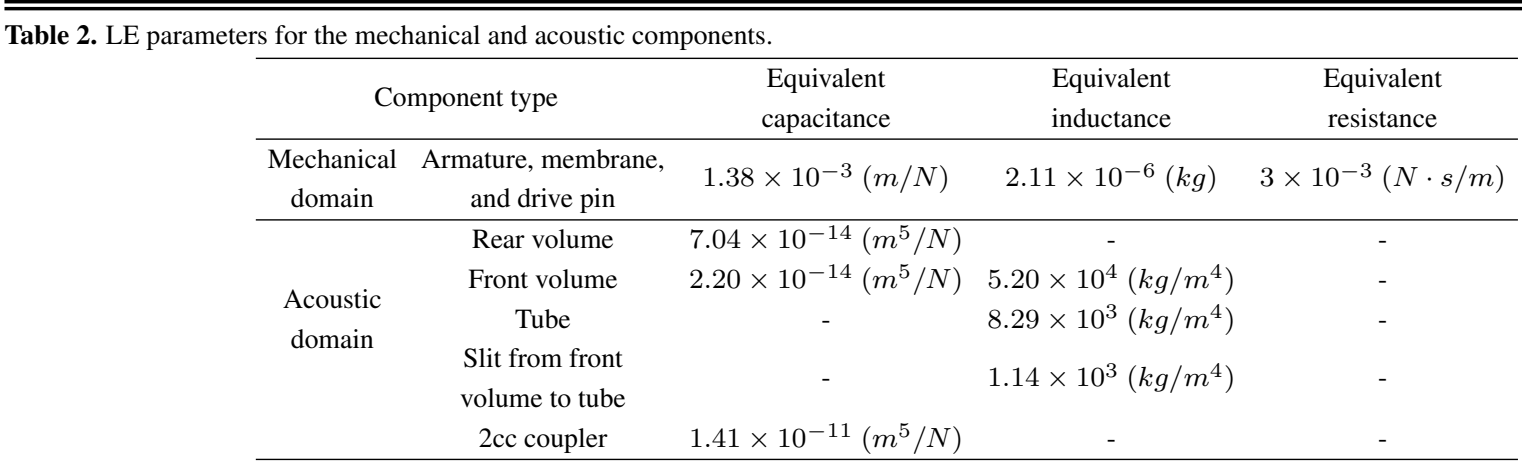

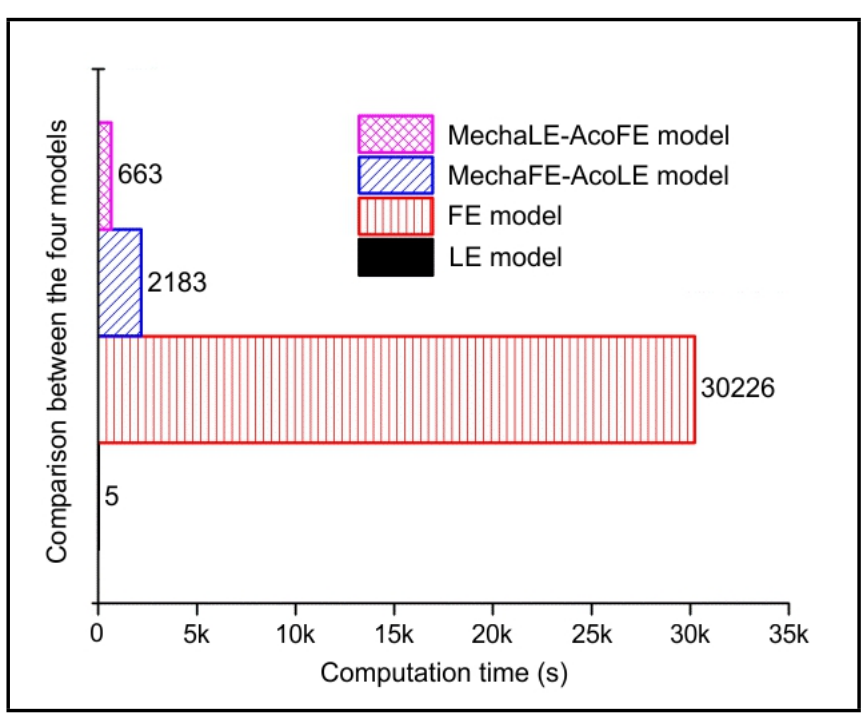

Figure 8. Comparison of the computation time between the concerned four models.

models. Finally, the two integrated models are compared to check their respective advantages and disadvantages.

\subsubsection{Comparison of the computation time between the concerned four models}

Besides the prescribed frequency resolution in Section 4.1.2, the meshing settings for the three FE involved models are also kept consistent, i.e. 12,053 elements for the mechanical domain and 23,490 elements for the acoustic domain. This aims at making the concerned four models comparable. A common personal computer with 4 cores $3.3 \mathrm{GHz}$ of CPU and $8 \mathrm{~GB}$ of RAM is used to perform the simulations.

Figure 8 clearly indicates that the two integrated models spend a little more time than the LE model but much less time than the full FE model. Therefore, it can be concluded that the proposed method is much more efficient than the full FE method.

\subsubsection{Evaluation of the integrated MechaFE-AcoLE model}

Figures 9(a) and 9(b) show the velocity response at MP and the SPL response in the 2cc coupler, respectively. For the velocity at MP, the MechaFE-AcoLE model is in substantial agreement with the FE model over the concerned frequency range, whereas the LE model is obviously not except a small range around the first peak. When it comes to the SPL response, the MechaFE-AcoLE model agrees well with the FE model up to $8 \mathrm{kHz}$, whereas the LE model is still obviously deviated from the FE model. The SPL discrepancy above $8 \mathrm{kHz}$ may be introduced by the LE modelled acoustic domain. Since wavelength of sound is getting closer to the dimensions of acoustic components with increase of frequency, the LE parameters are no longer applicable in the high frequency range. Figure 9(c) further demonstrates their respective SPL errors relative to the FE model. For the frequency up to $8 \mathrm{kHz}$, the error from the MechaFE-AcoLE model is mostly far less than $1 \mathrm{~dB}$ whereas that from the LE model is mostly bigger than $4 \mathrm{~dB}$. The latter is apparently unacceptable in engineering applications. It is the absence of higher-order modes of the mechanical domain for the LE model that leads to its remarkable deviation from the FE model.

From Figs. 9(a) and 9(b), we also notice that the SPL response from the LE model is obviously higher than the other two models in the frequency range before the first peak despite its smaller velocity. This seems irrational, but it does make sense when referring to the first two key vibration modes of the mechanical domain (Fig. 10). For the forced vibration system, the two modes additively contribute to the velocity at MP, whereas the volume velocity contributed by the fundamental mode is evidently subtracted by the second mode because of its concave bending shape. This results in the underestimated velocity at MP and the overestimated SPL output in the 2cc coupler from the LE model. The two modes correspond to the two peaks in the frequency responses in Fig. 9, respectively. The difference of the resonant frequencies here with that in Fig. 9 is caused by the acoustic loads in the system.

It is now evident that the integrated MechaFE-AcoLE model is much more reliable than the LE model. The conventional LE model for the example in Fig. 7 cannot even predict well the system in the frequency range before and around the fundamental resonant frequency. Therefore, we also hold that the developed MechaFE-AcoLE model is more adaptive.

\subsubsection{Evaluation of the integrated MechaLE-AcoFE model}

The above MechaFE-AcoLE model exhibits better prediction on the overall performance of BAR. Subsequently, we try to check if the integrated MechaLE-AcoFE model could make an improvement by modelling the acoustic domain with the FE method.

It is observed from Fig. 11 that the MechaLE-AcoFE model is not better than the LE model in evaluating the velocity at MP. The SPL response in the $2 \mathrm{cc}$ coupler roughly agrees with that from the FE model only around the last peak, which attributes 


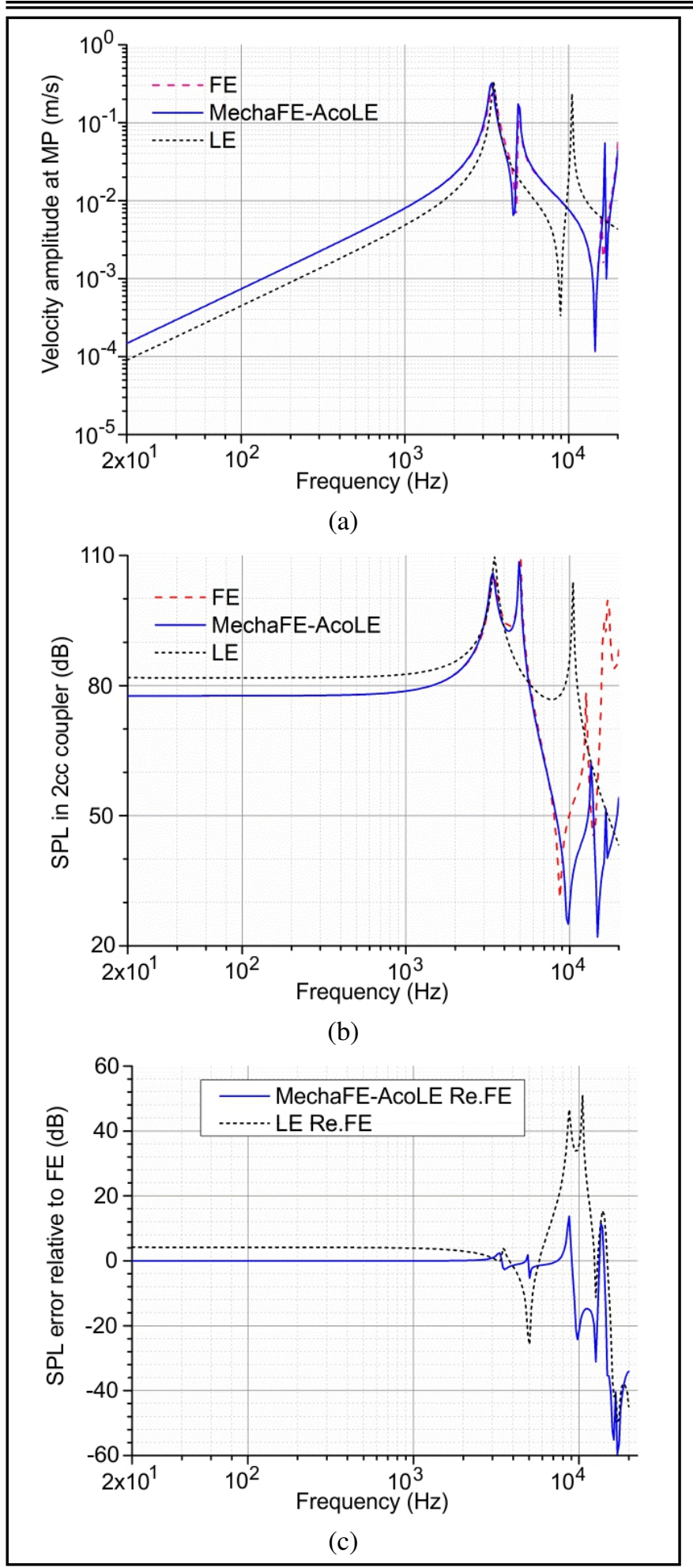

Figure 9. Frequency responses from the MechaFE-AcoLE model: (a) velocity response at MP; (b) SPL response in the 2cc coupler; (c) SPL errors relative to the FE model.

to the included higher-order modes of the acoustic domain. As a whole, this integrated model can just make a very limited improvement for the exemplified BAR model.

\subsubsection{SPL comparison between the concerned four models}

Figure 12 indicates the SPL responses in the 2cc coupler for all the concerned four models. Obviously, the two integrated models are the compromised results between the FE and LE

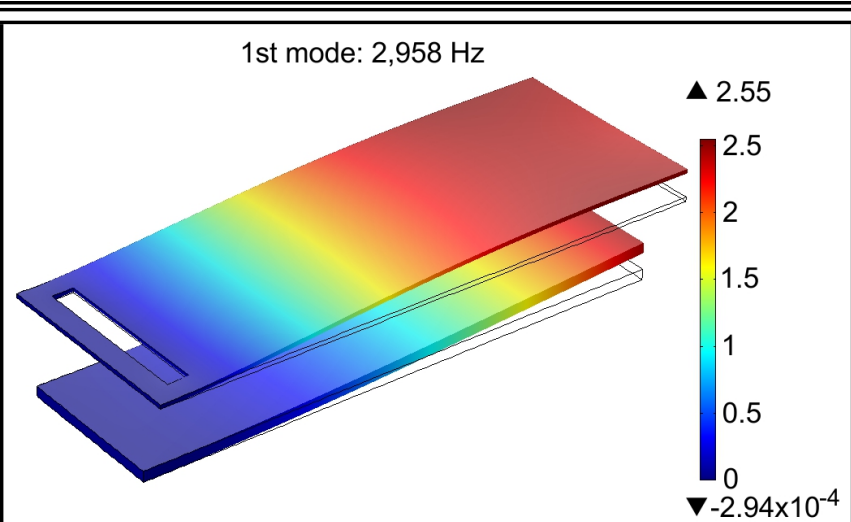

(a)

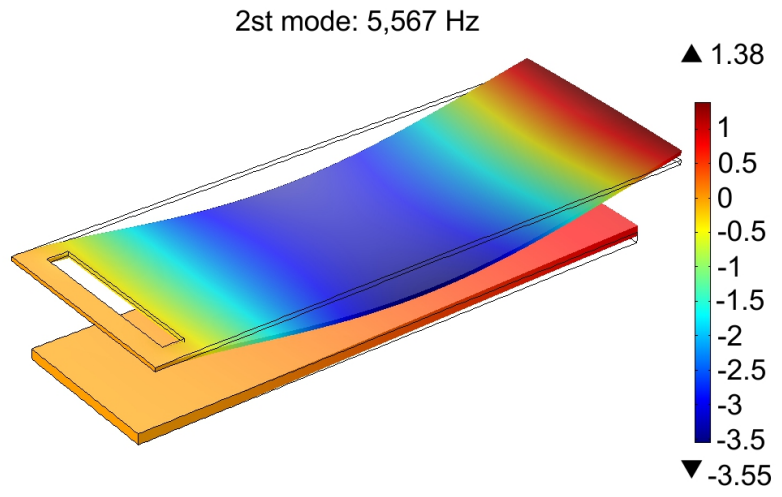

(b)

Figure 10. First two modes of the mechanical system of BAR: (a) fundamental mode (first mode); (b) second mode.

models. The overall performance of the exemplified BAR is dominated by the mechanical domain in the main audio frequency range, and by the acoustic domain merely in the high frequency range around the last peak. The transitional range around the third peak is determined by the interaction between the two domains. By comparing the two integrated models, the MechaFE-AcoLE model is preferentially suggested in the engineering application because of the better prediction of the system in the main audio frequency range.

To sum up, the two integrated FE-LE models, especially the MechaFE-AcoLE model, are far superior to the LE model in accuracy and to the FE model in efficiency.

\section{CONCLUSIONS}

As having the advantages of both the FE and LE methods, the proposed integrated FE-LE modelling method is much more accurate than the LE method and more efficient than the FE method. Specifically, the MechaFE-AcoLE model is more applicable in the practical development of BAR whereas the MechaLE-AcoFE model could be an auxiliary reference. Generally, the selection of the combination for the FE-LE modelling method depends on the interested point in application. Furthermore, the proposed method can be also applicable for developing other types of transducers incorporating multiphysics domains.

In this study, we merely create the models for a simplified BAR without the electromagnetic domain. Therefore, improvement can be made by considering the entire BAR model. 


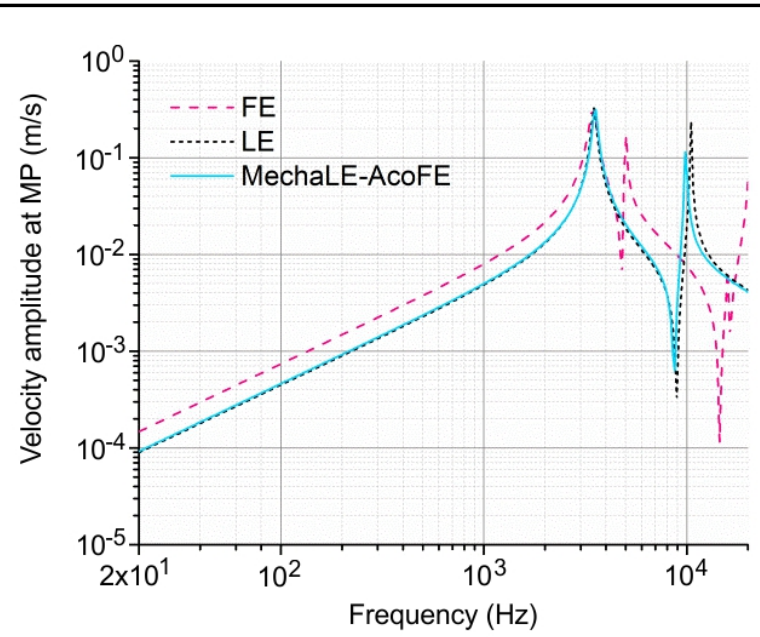

(a)

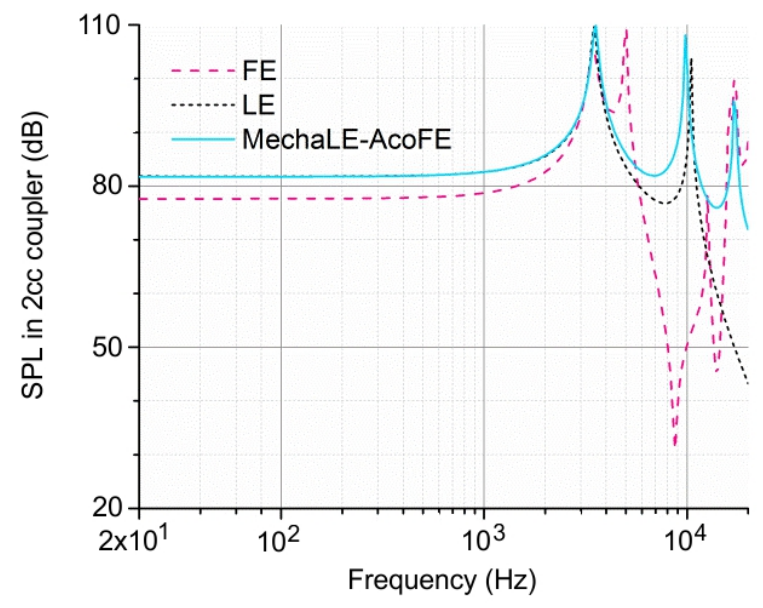

(b)

Figure 11. Frequency responses from MechaLE-AcoFE model: (a) velocity response at MP; (b) SPL response in the 2cc coupler.

For more physical domains contained, there are quite a few combinations. As a rule of thumb, the most interested domain should be preferentially modelled with the FE method whereas the others with the LE method. Besides, the improvement can also be obtained by including visco-thermal effects of the acoustic domain.

\section{REFERENCES}

1 Hunt, F. V. Electroacoustics: The analysis of transduction, and its historical background. Acoustical Society of America, New York, 213-235, (1954).

2 Madsen, H. S. Small balanced armature receiver for electronic telephone sets, Journal of the Audio Engineering Society, 19 (3), 209-212, (1971).

3 Kampinga, W. R., Bosschaart, C., Wijnant, Y. H. and Boer, A. D. The coupling of a hearing aid loudspeaker membrane to visco-thermal air layers, $14^{\text {th }}$ International Congress on Sound and Vibration, Cairns, Australia, (2007).

4 Jensen, J., Agerkvist, F. T. and Harte, J. M. Nonlinear timedomain modelling of balanced-armature receivers, Journal of the Audio Engineering Society, 59 (3), 91-101, (2011).

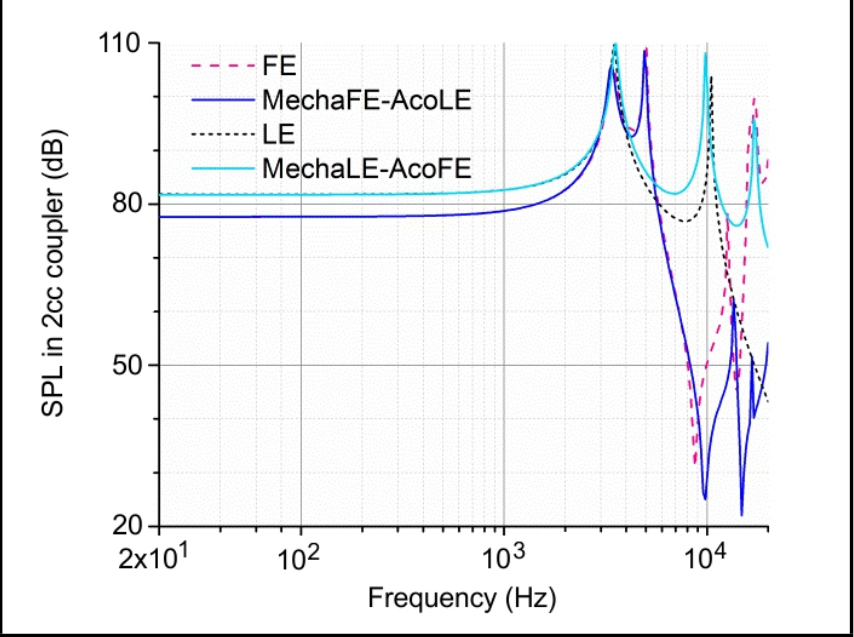

Figure 12. SPL comparison between the concerned four models.

5 Kim, N. and Allen, J. B. Two-port network analysis and modelling of a balanced armature receiver, Hearing Research, 301 (1), 156-167, (2013). https://dx.doi.org/10.1016/j.heares.2013.02.007

6 Tsai, Y. T. and Huang, J. H. A study of nonlinear harmonic distortion in a balanced armature actuator with asymmetrical magnetic flux, Sensors and Actuators A, 203 (6), 324334, (2013). https://dx.doi.org/10.1016/j.sna.2013.08.046

7 Jensen, J. Nonlinear distortion mechanisms and efficiency of miniature balanced-armature loudspeakers, $\mathrm{PhD}$ thesis, Technical University of Denmark, Denmark, (2014).

8 Bai, M. R., Liu, C. Y., and Chen, R. L. Optimization of microspeaker diaphragm pattern using combined finite element-lumped parameter models, IEEE Transactions on Magnetics, 44 (8), 2049-2057, (2008). https://dx.doi.org/10.1109/TMAG.2008.923316

9 Rashedin, R. and Meydan, T. Solenoid actuator for loudspeaker application, Sensors and Actuators A, 129 (1-2), 220-223, (2006). https://dx.doi.org/10.1016/j.sna.2005.11.049

10 Nguyen, C. H. and Pietrzko, S. J. Vibroacoustic FE analysis of an adaptive plate with PZT actuator/sensor pairs connected to a multiple-mode, electric shunt system, $\mathrm{Fi}$ nite Elements in Analysis and Design, 43 (15), 1120-1134, (2007). https://dx.doi.org/10.1016/j.finel.2007.08.011

11 Riley, K. F., Hobson, M. P., and Bence, S. J. Mathematical methods for physics and engineering: A comprehensive guide, $2^{\text {nd }}$ edn., Cambridge University Press, New York, 635, (2002).

12 Comsol Multiphysics 3.5a, Acoutics module users guide, 78, (2008).

13 William, W. T. and Dahleh, M. D. Theory of vibration with applications, $5^{\text {th }}$ edn., Prentice Hall, New Jersey, 273-274, (1998).

14 Raichel, D. R. The science and applications of acoustics, $2^{\text {nd }}$ edn., Springer, New York, 145, 153-155,134, (2006). 